\title{
On the Experimental Determination of Poisson's Ratio for Intact Rocks and Its Variation as Deformation Develops
}

\author{
Lu Dong, Hongfa Xu, Pengxian Fan $(\mathbb{D}$, and Zhichou Wu \\ State Key Laboratory of Disaster Prevention and Mitigation of Explosion and Impact, Army Engineering University of PLA, \\ Nanjing 210007, China \\ Correspondence should be addressed to Pengxian Fan; fan-px@139.com
}

Received 14 August 2020; Revised 23 December 2020; Accepted 18 January 2021; Published 4 February 2021

Academic Editor: Fengqiang Gong

Copyright $\odot 2021$ Lu Dong et al. This is an open access article distributed under the Creative Commons Attribution License, which permits unrestricted use, distribution, and reproduction in any medium, provided the original work is properly cited.

Poisson's ratio is of crucial importance for the theoretical and numerical analysis of rock engineering. It is an elastic parameter of the material and the ratio of the absolute value of lateral strain and axial strain when the material is under uniaxial tension or compression. However, it was rarely investigated compared with deformation modulus and strength. Rock materials are different from metal materials. The pure elastic deformation stage is usually very short or nonexistent in the process of uniaxial tension or compression. In this paper, in order to explore the behavior of Poisson's ratio, uniaxial compression tests according to The International Society for Rock Mechanics and Rock Engineering are performed on standard specimens of granite, marble, red sandstone, carbonate rock, coral concrete, etc. According to the results, Poisson's ratio, both the secant Poisson's ratio and tangent Poisson's ratio, increase with the externally applied stress. Therefore, regarding it as an elastic constant is worthy of a second thought. If the midpoint of the stress interval is fixed in the $50 \%$ of uniaxial compressive strength, the average Poisson's ratio is almost impervious to the varying span of the stress interval. In addition, the average Poisson's ratio is immune to the nonlinear deformation in the early loading stage. Thus, the average Poisson's ratio is a better index than the secant Poisson's ratio in describing the relationship between axial and lateral strains of hard rocks. The determination of Poisson's ratio of soft rocks needs further investigation because Poisson's ratio tends to exceed the theoretical limit in relatively low stress levels. The proposed viewpoint provides a deeper insight into the testing, determining, and using of Poisson's ratio.

\section{Introduction}

The International Society for Rock Mechanics and Rock Engineering (ISRM) defines Poisson's ratio as "the ratio of the shortening in the transverse direction to the elongation in the direction of applied force in a body under tension below the proportional limit" [1]. This definition of Poisson's ratio leaves much to be desired because it is extremely difficult to perform tensile tests on rock materials. Furthermore, the vast majority of surrounding rocks in rock engineering projects are not positioned in tensile stress states. As a result, none of the testing methods recommended by the ISRM, American Society for Testing and Materials (ASTM), or China's national standards use this definition of Poisson's ratio.

Poisson's ratio is a critical parameter in theoretical analyses and numerical simulations. However, there are few reports regarding its testing, valuation, and scope of application. In practice, engineers and researchers often use a fixed constant to describe the relationship between the orthogonal deformations of rocks under some force, without fully realizing the importance of scientifically determining Poisson's ratio to describe rock deformation behaviors. An in-depth understanding of Poisson's ratio will enable researchers to conduct better laboratory and in situ tests and deepen their understanding of the processes of rock (mass) deformation.

\section{Commonly Used Testing Methods}

Poisson's ratio is an elasticity parameter named after Siméon Denis Poisson (1781-1840). In 1828, Poisson proposed that [2] an isotropic elastic cylindrical rod of length $l$ and radius $r$ that is subjected to simple tensile stress experiences a shrinkage of $r(1-0.25 \delta)$ in the radial direction when its 
length increases by $l(1+\delta)$. In other words, the deformation parameter that was later named as Poisson's ratio was, at the time, thought to be a constant equal to 0.25 . It was only in 1859 that Kirchhoff demonstrated that Poisson's ratio is a nonconstant that depends on material properties, through experimental measurements of Poisson's ratio in steel and brass [3]. The long-held belief that Poisson's ratio is a simple constant was finally overturned by the efforts of SaintVenant and Voigt. Finally, in 1908, Gruneisen used direct tensile tests to measure the ratio between lateral and axial strains, thus determining Poisson's coefficient for the first time [4]. This method subsequently became widely used, and it is still in use today. Poisson's ratio varies only slightly from the initial value given by Poisson (0.25) in most metallic materials, and the methods for testing Poisson's ratio in these materials have also matured. However, it is much more difficult to test for Poisson's ratio in rock materials.

\subsection{The ISRM-Recommended Method. Although the ISRM} uses the traditional definition of Poisson's ratio, its recommended testing method [5] uses uniaxial compression tests to determine the numerical value of Poisson's ratio. In these tests, a standard rock specimen is either continuously loaded on a testing machine so that it fractures within $5-10 \mathrm{~min}$ or loaded at a rate of $0.5-1.0 \mathrm{MPa} / \mathrm{s}$. The axial and radial deformations or strain of the specimen are continuously monitored to obtain its stress-strain curve.

After the stress-strain curve of the specimen has been obtained, its Poisson's ratio, $\mu$, is calculated by

$$
\begin{aligned}
\mu & =\frac{\text { slope of axial stress }- \text { strain curve }}{\text { slope of diametric stress - strain curve }}, \\
& =\frac{E}{\text { slope of diametric stress - strain curve }},
\end{aligned}
$$

where $E$ is the axial deformation modulus (Young's modulus/elastic modulus).

Because of the nonlinearity of the stress-strain curve, Poisson's ratio may be divided into the tangent, average, and secant Poisson's ratios; the definitions of these ratios relate to their corresponding Young's moduli. The tangent Poisson's ratio is the slope of the stress-strain curve at a specific level of stress, and the average Poisson's ratio is the ratio between the differences in radial and tangential strain that correspond to the upper and lower limits of some interval of stresses. The secant Poisson's ratio is the slope of a line drawn from the origin that intersects the stress-strain curve at a specific level of stress.

The ISRM-recommended method has not been updated for more than 40 years. The 2014 update of the ASTMrecommended method [6] essentially adopted the concepts, testing procedures, and calculations of the ISRM method.

2.2. The Recommended Method of the Standard for Test Methods of Engineering Rock Mass. The Standard for Test Methods of Engineering Rock Mass (GB/T50266-2013) [7] recommends the use of uniaxial compression tests to determine Poisson's ratio of rocks. After the standard specimen's stress-strain curve is obtained, Poisson's ratio is calculated by substituting the axial and radial strain data into their corresponding equations.

The average Poisson's ratio for rocks $\mu_{a v}$ is given by

$$
\mu_{a v}=\frac{\varepsilon_{d b}-\varepsilon_{d a}}{\varepsilon_{l b}-\varepsilon_{l a}} .
$$

In equation (2), $\varepsilon_{d a}$ and $\varepsilon_{d b}$ are the radial strains that correspond to axial stresses $\sigma_{a}$ and $\sigma_{b} ; \varepsilon_{l a}$ and $\varepsilon_{l b}$ are the axial strains that correspond to $\sigma_{a}$ and $\sigma_{b}$, respectively.

The secant Poisson's ratio for rocks is given by

$$
\mu_{50}=\frac{\varepsilon_{d 50}}{\varepsilon_{l 50}}
$$

where $\varepsilon_{d 50}$ and $\varepsilon_{l 50}$ are the radial and hoop strains that correspond to an axial stress equal to $50 \%$ of the uniaxial compressive strength (UCS).

From equation (3), it may be observed that the secant Poisson's ratio in the Standard for Test Methods of Engineering Rock Mass uses 50\% of UCS as the reference point on the stress-strain curve. Therefore, it is a quite narrow definition of the secant Poisson's ratio.

Unless specified otherwise, all subsequent mentions of the secant Poisson's ratio in this paper use the definition of the ISRM-recommended method.

2.3. Poisson's Ratios of Intact Rocks. The range of Poisson's ratios for a variety of common rocks is shown in Figure 1 [8]. Here, it may be observed that most rocks exhibit a wide range of Poisson's ratios, e.g., $0.10-0.40$ for conglomerate, 0.10-0.33 for limestone, $0.05-0.30$ for rock salt, and $0.05-0.40$ for sandstone. Even marble, which is relatively homogenous in composition, has a Poisson's ratio range where the upper limit $(0.30)$ is twice the lower limit $(0.15)$.

The large range of Poisson's ratios in rocks is partly caused by significant heterogeneities in their geological history, mineral composition, crystallization, or depositional structure. However, it is also unclear whether Poisson's ratios provided in the literature are average or secant Poisson's ratios. As such, the methods by which these ratios were tested and calculated may have affected the reported Poisson's ratios.

\section{Tests and Calculations for Poisson's Ratio and Its Trends}

3.1. Stagewise Character of Intact Rock Deformations and Its Features. Studies have shown that most rocks have a progressive failure process, which can be divided into multiple stages with distinct stress-strain features (i.e., axial strain, lateral strain, relationship between these strains, and their trends) [9-11]: the crack closure stage, linear elastic deformation stage, crack initiation plus stable crack propagation, and unstable crack propagation.

The typical stress-strain curves of some red sandstone specimens from uniaxial compression testing are shown in Figure 2 [12]. Because it is difficult to accurately distinguish 
each stage of deformation in an axial deformation curve, it is necessary to use processed data such as the axial deformation modulus and crack volumetric strain [13]. The crack volumetric strain inside a specimen can be calculated by subtracting the elastic volumetric strain from the actual volumetric strain [14]. To calculate the elastic volumetric strain, one must use Poisson's ratio.

By processing the axial deformation data, one then obtains the axial deformation modulus plot (see Figure 3). In Figure 3, it is shown that the changes of the axial deformation modulus can be divided into three distinct stages: a rising segment, stable segment, and falling segment. The upper and lower stress limits of the stable segment correspond to the crack damage stress $\sigma_{c d}$ and crack closure stress $\sigma_{c c}$, and this segment is usually thought to correspond to the elastic stage. Because of there is no standard definition for $\sigma_{c c}$, the crack initiation stress $\sigma_{c i}$ is often used as the lower limit of the linear segment instead of $\sigma_{c c}$. This segment of the stress-axial strain curve is approximately linear, and it is typically used to define the elastic modulus.

$\sigma_{c i}$ of rock materials is approximately $30-50 \%$ of their UCS values [15], and $\sigma_{c d}$ of red sandstone is $60-80 \%$ of its UCS [12]. On the basis of this perspective, it is reasonable to choose the stress-strain curve around $40-60 \%$ of UCS to calculate the average elastic modulus of rocks, for the vast majority of rock types.

The ISRM-recommended method does not recommend a reasonable interval to calculate the average Poisson's ratio because it assumes that it can be taken from the method for determining Young's modulus. The Standard for Test Methods of Engineering Rock Mass also does not specify the upper and lower limits of the stress-strain curve that should be used to calculate the average Poisson's ratio. This lack of guidance makes it difficult to perform horizontal comparisons between Poisson's ratios reported by different researchers.

3.2. Strain Curve and Calculation of Poisson's Ratio. As compared to parameters such as Young's modulus and compressive strength, studies on the behavior of Poisson's ratio in each stage of rock deformation are relatively scarce. Walsh [16] found that opening and closing of rock cracks lead to the changes of Poisson's ratio. Therefore, Poisson's ratio is not constant and continuously increases by the uniaxial compression test. You and Hua [17] noted that it is far more difficult to determine Poisson's ratio using its original definition than it is to determine the elastic modulus because the "tangent Poisson's ratio" is almost always increasing; likewise, it is also difficult to precisely select the secant Poisson's ratio. Tu and Yang [18] reported that Grade III biotite dolomite specimens exhibit negative Poisson's ratios at low axial pressures during the initial compaction stage and then positive Poisson's ratios with further increases in axial stress. By conducting experiments on three types of rocks, Yu et al. [19] found that the tangent Poisson's ratio of rocks increases with compressive stress under compressive conditions and decreases with increasing tensile stress under tensile conditions. Recently, Kumar et al.
[20] investigated the influence of the unconfined compressive strength on Poisson's ratio. In the test, Poisson's ratio increases linearly in case of increasing uniaxial compressive strength. Xing et al. [21] discussed the evolution of Poisson's ratio at different strain rates, and it proved that there is no pure elastic stage where Poisson's ratio should be rate independent. By the results of Davarpanah et al. [22], it was also obtained that Poisson's ratio is highly decided by the stress. Benedek et al. [23] assumed that Poisson's ratio depends on the rigidity of the intact rock-increasing the brittleness of the rock material the Poisson's ratio should be decreasing.

In order to explore the evolution rule of Poisson's ratio, we performed uniaxial compression tests on standard specimens of granite, marble, red sandstone, carbonate rock, and coral concrete. These specimens were processed into cylinder samples after cutting and grinding, with a diameter of $50 \mathrm{~mm}$ and height of $100 \mathrm{~mm}$ (Figure 4). The MTS647.250 electro-hydraulic servo-controlled material test system was adopted to perform the uniaxial compression tests. The radial deformation and axial deformation were measured by MTS extensometers in Figure 5. The displacement control mode was set at a constant strain rate of $1 \times 10^{-5} \mathrm{~s}^{-1}$. PTFE films were placed on the contact position between specimen and two platens to diminish the end effect.

The equations for calculating tangent Poisson's ratio $\mu_{t}$, average Poisson's ratio $\mu_{a v}$, and secant Poisson's ratio $\mu_{s}$ are shown in Figure 6, and it is noted that the radial strains were treated as positive values to make it easier to plot their values in this four axial strain-radial strain curves.

In these experimental data, it may be observed that radial strain always increases more quickly than axial strain throughout the deformation process. As a result, all of the plots curve upwards. Furthermore, their Poisson's ratios increase monotonously without exhibiting a distinct plateau region. This behavior is completely different from that of the elastic modulus. Furthermore, because of the convexity of the axial strain-lateral strain curve, $\mu_{s}$ is usually smaller than $\mu_{t}$ and $\mu_{a v}$. Between the three Poisson's ratios, it is $\mu_{t}$ that grows most quickly with stress.

3.3. Secant Poisson's Ratio. To examine the behavior of the secant Poisson's ratio, the origin of the secant was fixed at the zero-stress point, but the reference point (point $C$ in Figure 6) was treated as a moving point that changes with stress. The secant Poisson's ratios that were calculated from our experimental data are shown in Figure 7.

In Figure 7, it is shown that the evolution of the secant Poisson's ratio can be divided into distinct stages. In the lowstress crack closure stage, the lateral strains are relatively small and unstable, and the growth in lateral strain depends mainly on the material characteristics rather than longitudinal deformation. The corresponding Poisson's ratios of this stage are small, slow-growing, and strongly fluctuating to the point where negative Poisson's ratios may arise during the initial stages of the loading process. When the stress increases to $20-30 \%$ of the peak strength, the secant Poisson's ratio then enters the linear growth stage, where it 


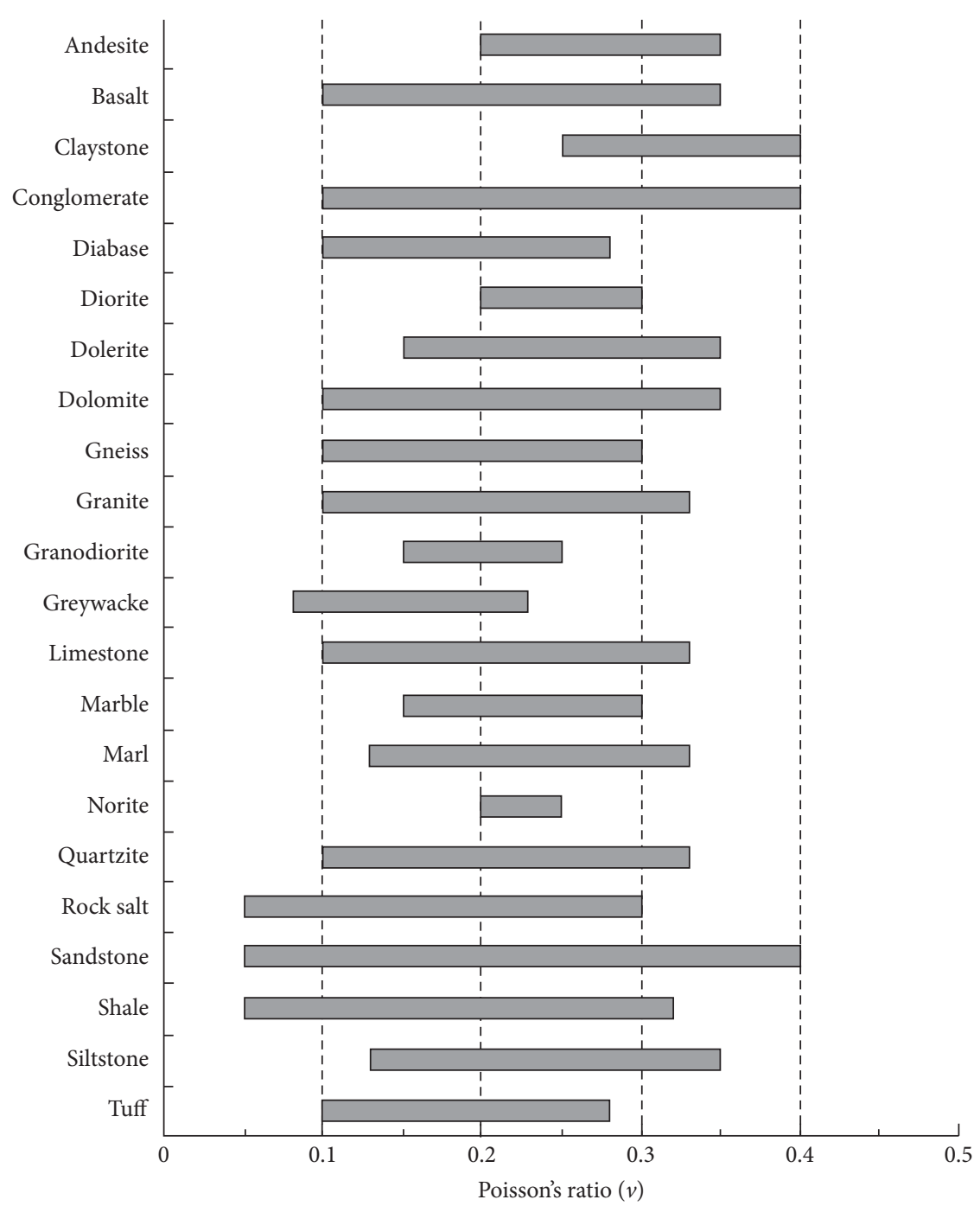

Figure 1: Distribution of Poisson's ratios for typical types of rocks [8].

increases in proportion to axial stress. When the stress increases to $70-80 \%$ of the peak strength, the secant Poisson's ratio grows in a rapid and nonlinear manner, and it exceeds the normal range of values for Poisson's ratio prior to failure.

The stress threshold where the secant Poisson's ratio transitions into the nonlinear rapid growth stage roughly corresponds to the failure stress. After the failure stress is reached, the axial deformation modulus decreases, but the crack propagation rate accelerates. This phenomenon induces a significant degree of lateral expansion, which greatly increases the calculated secant Poisson's ratio.

3.4. Tangent Poisson's Ratio. In theory, the tangent Poisson's ratio is the tangential slope of the axial strain-radial strain curve. In practice, however, this curve consists of a series of scattered points. As compared to the secant Poisson's ratio, the calculation of the tangent Poisson's ratio is more sensitive to variations in the testing method and sampling frequency. When one computes the tangent Poisson's ratio, it is necessary to determine the $\Delta \varepsilon$ sampling interval, which is usually determined by the desired level of stress. For example, to determine the tangent Poisson's ratio at $50 \%$ of UCS, one must use the strain that corresponds to this level of stress as the center point of the stress-sampling interval (henceforth referred to as the "stress interval"). The span of the stress interval directly affects the calculated value of the tangent Poisson's ratio and the shape of its curve.

In Figure 8, the variation in the tangent Poisson's ratio with stress interval width is shown for two different rocks. Here, it is shown that the calculated tangent Poisson's ratios exhibit intense fluctuations when the width of the stress interval is roughly $1 \%$ of UCS. Increasing the span of the stress interval reduces the fluctuations of the tangent Poisson's ratio; the tangent Poisson's ratio becomes stable only when the stress interval is 5\% of UCS or larger. From the testing perspective, the tangent Poisson's ratio is essentially the average Poisson's ratio taken over a narrow stress interval, and there is a great deal of uncertainty in how it is calculated.

By reducing the sampling frequency, it is possible to suppress fluctuations by discarding some information. 


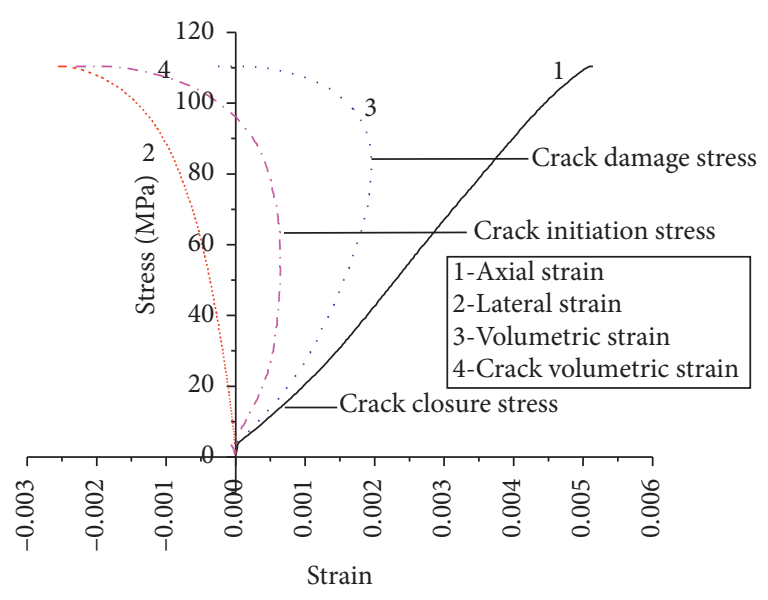

FIgURe 2: Typtical stress-strain curves of red sandstone specimens [12].

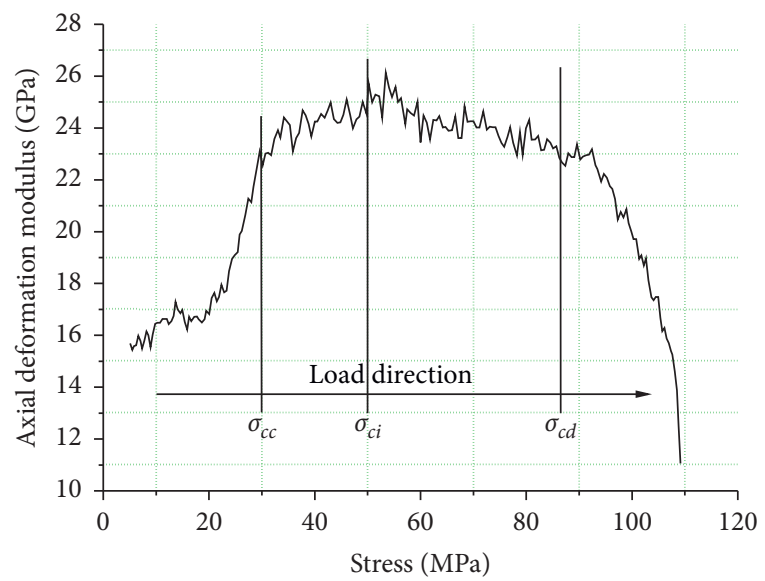

Figure 3: The typical axial deformation modulus curve of a red sandstone specimen.

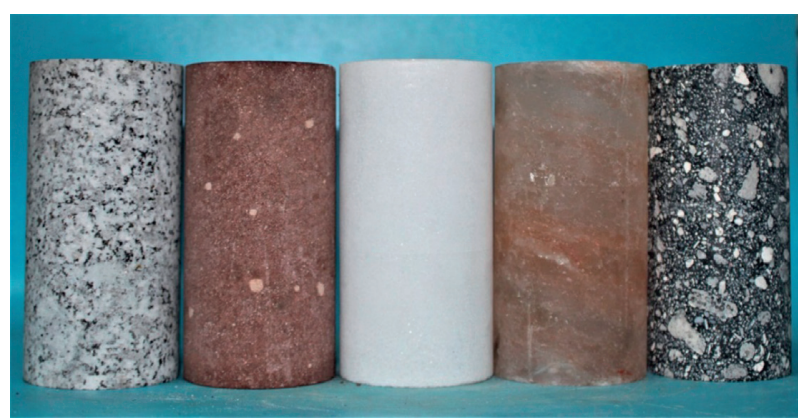

FIgURE 4: Main types of standard specimens.

Alternatively, one may process the strain data using smoothing methods (moving averages or least-squares fitting) to smooth the variations of the tangent Poisson's ratio plot. Nonetheless, the calculation of the tangent Poisson's ratio always involves artificial preprocessing and data selection processes, and different preprocessing methods inevitably lead to different values, thus impacting the

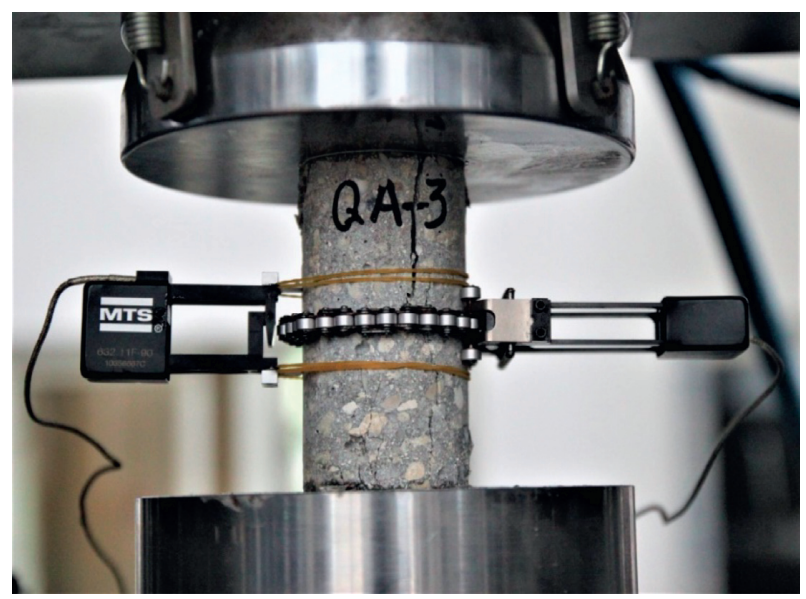

Figure 5: A specimen in the uniaxial compression tests.

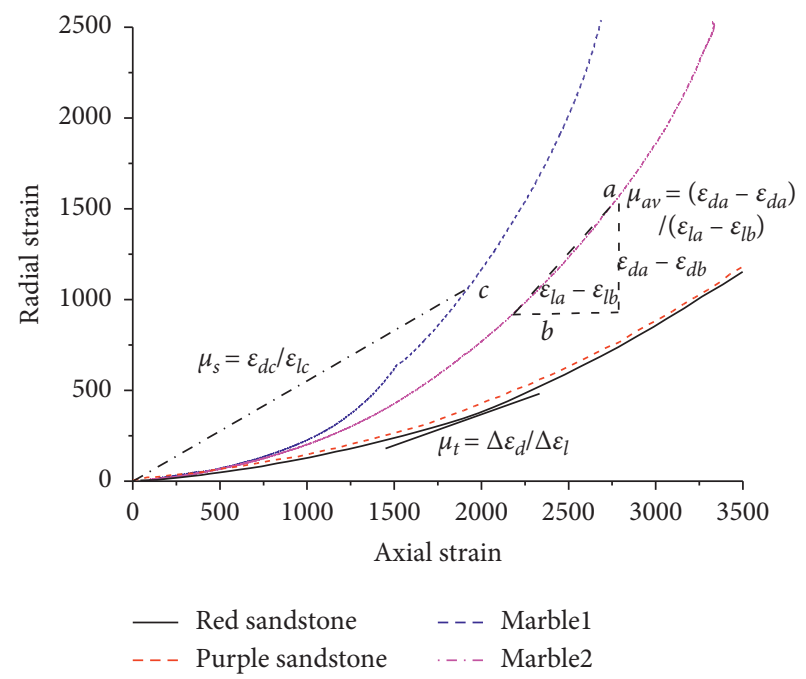

Figure 6: The equations for calculating $\mu_{t}, \mu_{a v}$, and $\mu_{s}$.

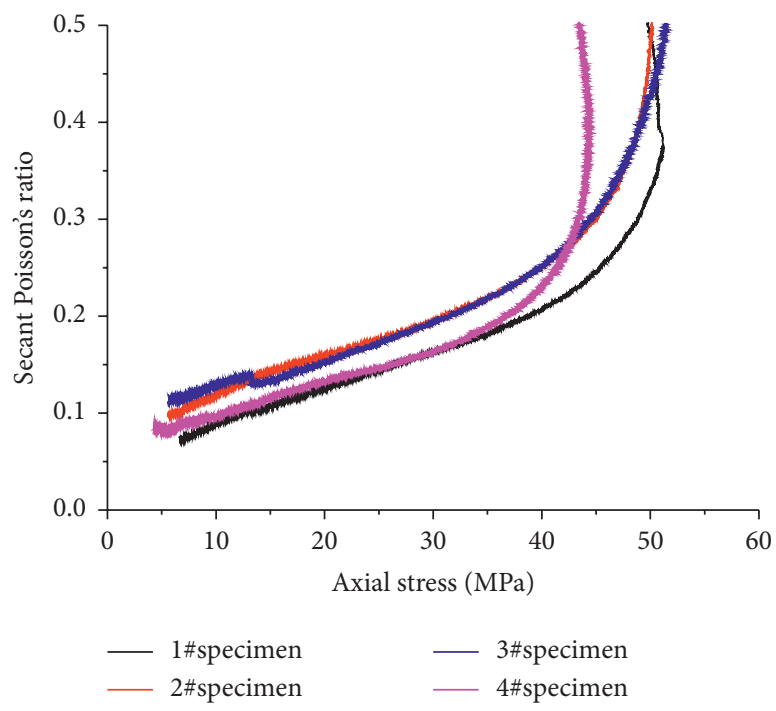

Figure 7: Secant Poisson's ratios of several marble specimens. 


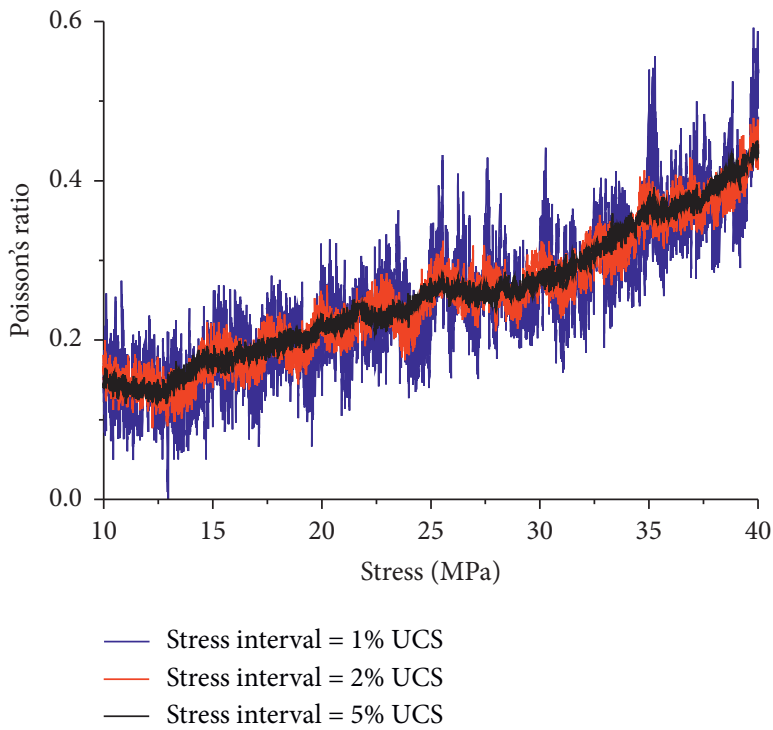

(a)

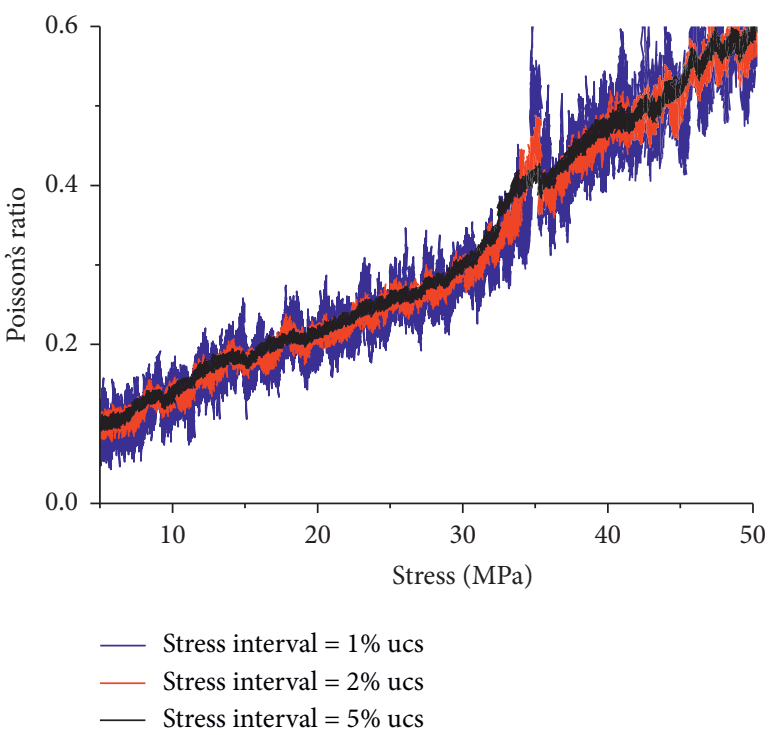

(b)

Figure 8: Tangent Poisson's ratios of two types of hard rocks with varying stress intervals. (a) Marble specimens. (b) Red sandstone specimens.

objectivity of this parameter. The uncertainty of the method and the resulting uncertainty of calculation inevitably produce adverse effects on the analysis of the gradual deformation process of the rock. Uncertainty and sensitivity analysis have recently received widespread interest of researchers in many fields. $\mathrm{Vu}-\mathrm{Bac}$ et al. [24] provided a sensitivity analysis toolbox for quantifying the influence of uncertain input parameters on uncertain model outputs which we plan to consider in research.

As a whole, the tangent and secant Poisson's ratios exhibit similar growth trends. Given a wide stress interval, the midsegment of the tangent Poisson's ratio plot grows almost linearly with stress. When the stress increases from $20 \%$ of UCS to $80 \%$ of UCS (approximately $40 \mathrm{MPa}$ ), the tangent Poisson's ratio of the marble specimen increases by more than $100 \%$. In a red sandstone specimen, it was observed that its tangent Poisson's ratio approached the extreme limit of reasonable Poisson's ratio values $(0.50)$ when the stress was $80 \%$ of UCS (approximately $45 \mathrm{MPa}$ ); this is more than twice its Poisson's ratio at $20 \%$ of UCS.

3.5. Average Poisson's Ratio. First, we examined the average Poisson's ratio with a fixed center point at $50 \%$ of UCS. The upper and lower limits were $50 \% \pm 5 \%(45-55 \%)$ of UCS, $50 \% \pm 10 \%(40-60 \%)$ of UCS, $50 \% \pm 15 \%$ (35-65\%) of UCS, $50 \% \pm 20 \%(30-70 \%)$ of UCS, and $50 \% \pm 25 \%(25-75 \%)$ of UCS. The average Poisson's ratios were calculated using equation (2), and the average Poisson's ratios of a few types of rocks are shown in Figure 9.

In Figure 9, it is shown that the average Poisson's ratio changes very little when the sampling interval is extended in a symmetric manner from $50 \%$ of UCS. The average Poisson's ratios of hard materials, such as sandstone, marble, and coral concrete, are relatively small, and their values remain within a reasonable range. However, soft and strongly dilatant rocks, such as loose tuff $(\mathrm{UCS}=24.08 \mathrm{MPa})$ and rock salt $(\mathrm{UCS}=33.37 \mathrm{MPa}$ ) become irreversibly damaged at low stress levels. Therefore, if one uses the recommended method to calculate their average Poisson's ratios (that is, by calculating their average Poisson's ratio at $50 \%$ of UCS), the resulting Poisson's ratios are often greater than the theoretical maximum. Therefore, it is not always appropriate to calculate the average Poisson's ratio of a rock at $50 \%$ of UCS.

We then calculated the average Poisson's ratios of a few types of rocks with a moving center point $(20 \%, 30 \%, 40 \%$, $50 \%, 60 \%$, and $70 \%$ of UCS) and a fixed stress interval width of $10 \%$ (i.e., the upper and lower limits are $\pm 5 \%$ from the center point), as shown in Figure 10. To facilitate comparisons, the secant Poisson's ratios of these materials are also plotted in Figure 10.

In Figure 10, it is shown that the average Poisson's ratio increases monotonously with stress, much like the secant Poisson's ratio.

In some hard rocks, the average Poisson's ratio can be more than $100 \%$ larger than the secant Poisson's ratio for the same reference point. In the $20-70 \%$ of the UCS range, red sandstone, marble, compacted tuff, and coral concrete have average Poisson's ratios that are 1.43-2.03, 1.17-2.00, $1.15-1.72$, and 1.46-1.94 times their secant Poisson's ratios, respectively.

The average Poisson's ratios of the two soft rocks remain below 0.5 only when the reference point is positioned at a low level of stress, and they exceed the reasonable range of 


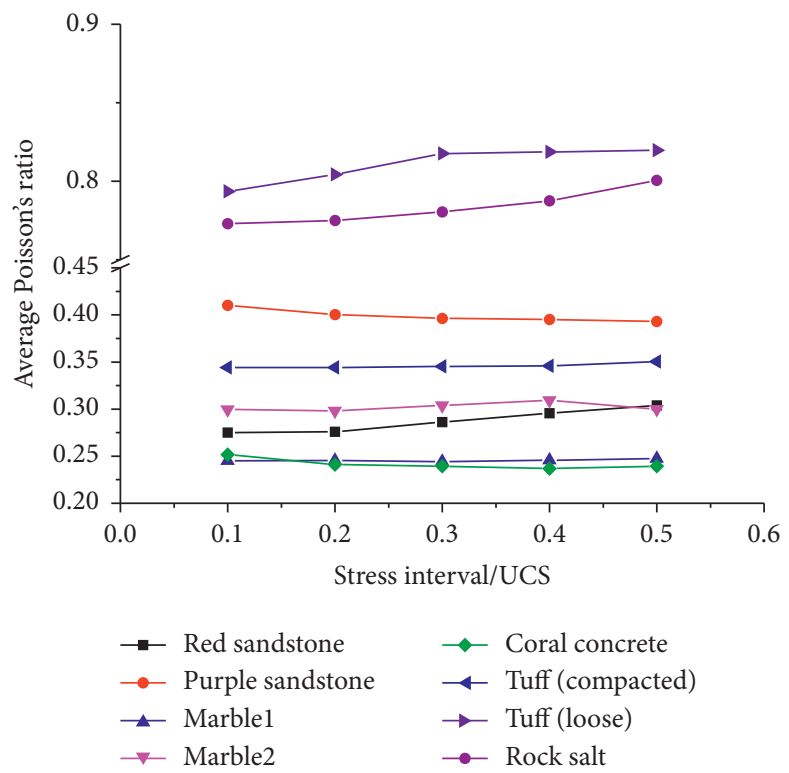

FIgURe 9: Average Poisson's ratios calculated with different stress intervals (with the center point being fixed at 50\% of UCS).

Poisson's ratio values even at small secant Poisson's ratios and $40-60 \%$ of UCS. Therefore, the secant, tangent, and average Poisson's ratios are meaningful only in a qualitative sense when characterizing the deformation processes of soft rocks.

\section{Discussion}

Poisson's ratio is the ratio between the absolute values of the lateral normal strain and longitudinal normal strain, when the material is uniaxially tensioned or compressed. It is an elastic constant that reflects the lateral deformation of a material. However, by analyzing the deformation processes of a wide variety of rocks under uniaxial compression, we found that Poisson's ratio and Young's modulus behave quite differently: there is no plateau in Poisson's ratio because the secant and average Poisson's ratios both increase monotonously with increasing stress. This behavior could be related to the irreversibility of compaction in the direction of compression and microcrack propagation in the principal stress direction.

By studying the progressive failure of granites, Eberhardt et al. [25] found that the lateral deformation modulus decreases monotonously with increasing stress (Figure 11), and the axial deformation modulus exhibits a distinct plateau during the linear elastic deformation stage.

According to equation (1), which was provided by the ISRM, Poisson's ratio is equal to the ratio between the axial and lateral deformation moduli. However, because Poisson's ratio of a rock changes continuously, even in the linear elastic deformation stage, it is a questionable practice to treat Poisson's ratio as an elastic constant. The value of Poisson's ratio can have a significant influence on how progressive failure processes are divided into stages because the crack propagation threshold can change by more than $40 \%$ for a \pm 0.05 change in Poisson's ratio [25].
In the current ISRM-recommended method's definitions of the secant, average, and tangent Poisson's ratios, the tangent Poisson's ratio is not actually different from the average Poisson's ratio for testing purposes, and the data processing steps required to calculate the tangent Poisson's ratio are susceptible to human error. Therefore, the Standard for Test Methods of Engineering Rock Mass has discarded the concept of tangent Poisson's ratios and provides testing methods only for the average Poisson's ratio and secant Poisson's ratio.

The generalized secant Poisson's ratio (where the secant's origin is fixed to the zero-stress point, while the second reference point is allowed to vary with stress) also increases monotonously with the stress level of the reference point, and it does not converge to some value. The Standard for Test Methods of Engineering Rock Mass narrowly defines the secant Poisson's ratio as the strain that corresponds to $50 \%$ of UCS, which gives it a uniquely defined value. Although this compromise makes it possible to compare Poisson's ratios of different materials, it does not allow this parameter to provide an accurate description of the relationship between axial and radial deformations. The secant Poisson's ratio includes the specimen's deformation data in the low-stress compaction stage. These values are generally quite small, which makes it susceptible to factors such as initial loading conditions of the test and zeroing of the displacement sensor.

The average Poisson's ratio reflects the relative change in axial and radial strain at the upper and lower limits of some stress interval. As the stress interval moves from the lowstress zone to the high-stress zone, the average Poisson's ratio also grows significantly; this growth is usually much greater than that of the secant Poisson's ratio. Because the average Poisson's ratio is sensitive to the choice of the stress interval, we suggest that the stress interval should be defined in the specifications of any updated standard. When the 


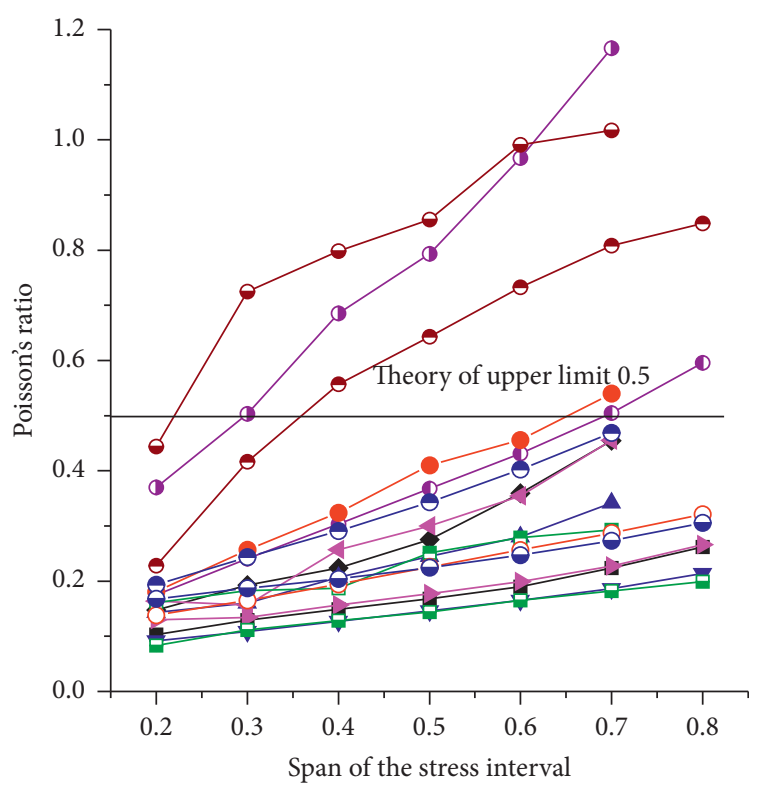

$$
\begin{array}{ll}
\text { Average Poisson's ratio } & \text { Secant Poisson's ratio } \\
- \text { Red sandstone } & - \text { Red sandstone } \\
- \text { - Purple sandstone } & - \text { Purple sandstone } \\
- \text { - Marble1 } & - \text { Marble1 } \\
- \text { Marble2 } & \rightarrow \text { Marble2 } \\
- \text { - Coral concrete } & - \text { Coral concrete } \\
- \text { Tuff (compacted) } & - \text { Tuff (compacted) } \\
- \text { - Tuff (loose) } & - \text { Tuff (loose) } \\
- \text { - Rock salt } & - \text { Rock salt }
\end{array}
$$

Figure 10: Average and secant Poisson's ratios with different reference points. The span of the stress interval for calculating the average Poisson's ratio was fixed at $10 \%$ of UCS.

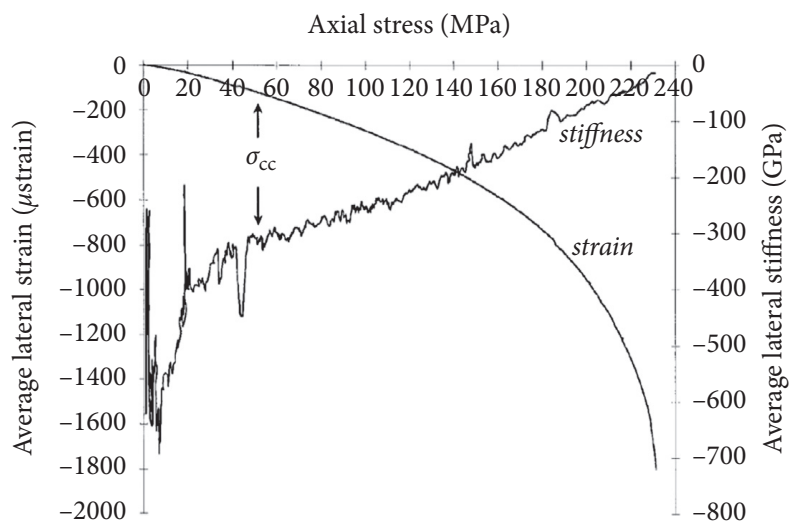

Figure 11: Lateral strain curve and lateral deformation stiffness of Canadian URLAE pink granite [25].

center point is fixed at $50 \%$ of UCS, the span of the stress interval does not significantly affect the calculated average Poisson's ratio. For hard rocks, this value is, in essence, the average relationship between axial and radial deformations in the linear elastic deformation stage. However, in soft and highly dilatant rocks, the significance of Poisson's ratio and its testing methods still require further investigation.

Furthermore, the average and secant Poisson's ratios tend to differ quite significantly; because Poisson's ratios provided by many reports do not specify how these ratios 
were calculated or tested, it is difficult to interpret and apply their experimental data.

\section{Conclusions}

The following conclusions were drawn by analyzing Poisson's ratios of several varieties of the rock:

(1) In rock materials, the tested Poisson's ratio is not a constant. It can be more accurately described as an elastic deformation parameter that monotonously increases with stress during the compressive processes.

(2) Different rocks exhibit different behaviors in their Poisson's ratios. In hard rocks, the secant and average Poisson's ratios grow approximately linearly with stress in the main stages of the loading process. However, in soft rocks, these ratios quickly increase beyond the theoretical maximum of 0.5 in the initial loading stage. As such, the method for determining Poisson's ratio in soft rocks requires further investigation.

(3) When the center point of the stress interval is set to $50 \%$ of UCS, the span of the stress interval has a negligible effect on the calculated average Poisson's ratio. Furthermore, this value is not affected by nonlinear deformations in the initial loading stage, and it is more suitable for describing the deformation behaviors of hard rocks than is the secant Poisson's ratio.

(4) Because the secant and average Poisson's ratios differ significantly, it is important to clarify how Poisson's ratio was obtained before applying these values.

Because of the immense variety of rocks in this world, which all have differing characteristics, our conclusions are not likely to be universally applicable because of the limited number of specimens that were included in this study. Many more empirical studies are needed to obtain a deep and thorough understanding of the behavior of Poisson's ratio in rocks.

\section{Data Availability}

The processed data required to reproduce the findings of this study cannot be shared at this time as they form part of an ongoing study. The data supporting the findings of this study are available from the corresponding author upon request.

\section{Conflicts of Interest}

The authors declare that they have no conflicts of interest.

\section{Acknowledgments}

This work was supported by the National Natural Science Funds of China (Grant nos. 51979280 and 51527810) and Natural Science Funds of Jiangsu Province (Grant no. BK20171400).

\section{References}

[1] S. P. Timoshenko, History of Strength of Materials, Dover Publications, New York, NY, USA, 1983.

[2] ISRM, ISRM Terminology (English, F., German), ISRM, Lizbon, Portugal, 1975.

[3] I. Todhunter and K. Pearson, A History of the Theory of Elasticity and of the Strength of Materials from Galilei to the Present Time, Cambridge University Press, Cambridge, UK, 1893.

[4] J. F. Bell, "The experimental foundations of solid mechanics," in Encyclopedia of Physics. Vol. VI a/1, Reprint of 1973 Original, S. Flugge, Ed., Springer, Berlin, Germany, 1984.

[5] Z. T. Bieniawski and M. J. Bernede, "Suggested methods for determining the uniaxial compressive strength and deformability of rock materials," International Journal of Rock Mechanics and Mining Sciences, vol. 16, pp. 135-140, 1978.

[6] American Society for Testing and Materials, "ASTM D7012$14 \mathrm{e} 1$ standard test methods for compressive strength and elastic moduli of intact rock core specimens under varying states of stress and temperatures," in Annual Book of ASTM StandardsAmerican Society for Testing and Materials, West Conshohocken, PA, USA, 2014.

[7] China Planning Press, GB/T50266-2013. Standard for Test Methods of Engineering Rock Mass, China Planning Press, Beijing, China, 2013, in Chinese.

[8] H. Gercek, "Poisson's ratio values for rocks," International Journal of Rock Mechanics and Mining Sciences, vol. 44, no. 1, pp. 1-13, 2007.

[9] Z. T. Bieniawski, "Stability concept of brittle fracture propagation in rock," Engineering Geology, vol. 2, no. 3, pp. 149-162, 1967.

[10] Z. T. Bieniawski, "Mechanism of brittle fracture of rock," International Journal of Rock Mechanics and Mining Sciences \& Geomechanics Abstracts, vol. 4, no. 4, pp. 395-406, 1967.

[11] C. D. Martin and N. A. Chandler, "The progressive fracture of Lac du Bonnet granite," International Journal of Rock Mechanics and Mining Sciences \& Geomechanics Abstracts, vol. 31, no. 6, pp. 643-659, 1994.

[12] H. Wang, P. Fan, M. Wang et al., "Influence of strain rate on progressive failure process and characteristic stresses of red sandstone," Rock and Soil Mechanics, vol. 32, no. 5, pp. 1340-1346, 2011, in Chinese.

[13] E. Eberhardt, Brittle Rock Fracture and Progressive Damage in Uniaxial Compression, University of Saskatchenwan, Saskatoon, Canada, 1998.

[14] C. D. Martin, Strength of Massive Lac du Bonnet Granite Around Underground Openings, University of Manitoba, Winnipeg, Canada, 1993.

[15] M. S. Diederichs, P. K. Kaiser, and E. Eberhardt, "Damage initiation and propagation in hard rock during tunnelling and the influence of near-face stress rotation," International Journal of Rock Mechanics and Mining Sciences, vol. 41, no. 5, pp. 785-812, 2004.

[16] J. B. Walsh, "The effect of cracks in rocks on Poisson's ratio," Journal of Geophysical Research, vol. 70, no. 20, pp. 52495257, 1965.

[17] M. You and A. Hua, "Determination of Poisson's ratio for rock material by experiment of confining pressure reduction," Journal of Experimental Mechanics, vol. 12, no. 2, pp. 274-278, 1997, in Chinese.

[18] Z. Tu and Q. Yang, “Test research on negative Poisson's ratio of rock mass," Rock and Soil Mechanics, vol. 29, no. 10, pp. 2833-2837, 2008, in Chinese. 
[19] X. Yu, Q. Wang, X. Li et al., "Experimental research on deformation of rocks in direct tension and compression," Rock and Soil Mechanics, vol. 29, no. 1, pp. 18-22, 2008, in Chinese.

[20] R. Kumar, K. Bhargava, and D. Choudhury, "Correlations of uniaxial compressive strength of rock mass with conventional strength properties through random number generation," International Journal of Geomechanics, vol. 17, no. 2, 2017.

[21] H. Z. Xing, Q. B. Zhang, and J. Zhao, "Stress thresholds of crack development and Poisson's ratio of rock material at high strain rate," Rock Mechanics and Rock Engineering, vol. 51, no. 3, pp. 945-951, 2017.

[22] M. Davarpanah, G. Somodi, L. Kovács, and B. Vásárhelyi, "Complex analysis of uniaxial compressive tests of the Mórágy granitic rock formation (Hungary)," Studia Geotechnica et Mechanica, vol. 41, no. 1, pp. 21-32, 2019.

[23] B. A. Lógó and B. Vásárhelyi, "Estimation of the Poisson's rate of the intact rock in the function of the rigidity," Periodica Polytechnica Civil Engineering, vol. 63, no. 4, pp. 1030-1037, 2019.

[24] N. Vu-Bac, T. Lahmer, X. Zhuang, T. Nguyen-Thoi, and T. Rabczuk, "A software framework for probabilistic sensitivity analysis for computationally expensive models," $A d$ vances in Engineering Software, vol. 100, pp. 19-31, 2016.

[25] E. Eberhardt, D. Stead, B. Stimpson, and R. S. Read, "Identifying crack initiation and propagation thresholds in brittle rock," Canadian Geotechnical Journal, vol. 35, no. 2, pp. 222-233, 1998. 